\title{
sciendo
}

Current Issues in Pharmacy and Medical Sciences

Formerly ANNALES UNIVERSITATIS MARIAE CURIE-SKLODOWSKA, SECTIO DDD, PHARMACIA

journal homepage: http://www.curipms.umlub.pl/

\section{The diagnostic value of immunohistochemical staining of the interstitial vascular C4d complement in membranous nephropathy}

\author{
Tala Pourlak ${ }^{1}$, Sonya Sharifi ${ }^{1,2}$, Sepideh Zununi Vahed ${ }^{2}$, \\ Elham Ahmadian $^{2 \star}$, Magali Cucchiarini ${ }^{3 *}$ (1) \\ ${ }^{1}$ Connective Tissue Diseases Research Center, Tabriz University of Medical Sciences, Tabriz, Iran \\ ${ }^{2}$ Kidney Research Center, Tabriz University of Medical Sciences, Tabriz, Iran \\ ${ }^{3}$ Center of Experimental Orthopaedics, Saarland University Medical Center, Homburg/Saar, Germany
}

\section{ARTICLE INFO \\ Received 12 May 2021 \\ Accepted 20 September 2021}

\section{Keywords:}

membranous

glomerulonephritis,

minimal change disease,

C4d immunohistochemical

staining,

immunofluorescence

microscopy.

\begin{abstract}
Membranous glomerulonephritis (MGN) is the most common cause of adulthood nephrotic syndrome. Diagnosis of membranous nephritis is based on light electron immunofluorescence microscopy and clinical signs. Immune complex deposition against podocyte antigens such as phospholipase A2 receptor (PLA2R) activates the complement system. Of this, complement Component $\mathrm{C} 4 \mathrm{~d}(\mathrm{C} 4 \mathrm{~d})$ is involved in the classical and lectin pathways. This marker may be used by immunohistochemistry to diagnose MGN when other methods are not available. In this work, C4d expression was monitored by immunohistochemical analysis in the glomerular capillaries of patients with primary MGN (study group, $\mathrm{N}=33$ ) versus patients with minimal change disease $(\mathrm{MCD}$, control group, $\mathrm{N}=20$ ) in a cross-sectional evaluation performed based on the diagnosis confirmed by light microscopy and immunofluorescence. There was no significant demographic difference between the two groups except for age $(\mathrm{P}=0.002)$. C4d immune-expression was positive in glomerular capillary (2+ to $4+)$ in most of the MGN patients, while it was negative in the MCD group. The sensitivity and specificity of C4d immunostaining were $95 \%$ and $100 \%$, respectively. The Pearson correlation coefficient was 0.74 between C4d (immunohistochemistry) and immunoglobulins (IgG; immunofluorescence) and 0.65 between $\mathrm{C} 4 \mathrm{~d}$ (immunohistochemistry) and the C3 complement product (immunofluorescence). Immunohistochemical evaluation of $\mathrm{C} 4 \mathrm{~d}$ is, therefore, a sensitive and specific method that has a high correlation with IgG immunofluorescence.
\end{abstract}

\section{INTRODUCTION}

Membranous glomerulonephritis (MGN) is among the well-known etiologies of nephrotic syndrome. It affects approximately $30 \%$ of all adults and $1-9 \%$ of the total pediatric population worldwide. The disease is more common in males, and the ages 30-50 exhibit the peak incidence. While primary MGN is more common, other pathologies such as systemic lupus erythematosus, tumors and infection can result in secondary MGN in $25 \%$ of all cases [1]. Patients with kidney failure at diagnosis, glomerulosclerosis, high rate of proteinuria, vascular damage and tubulointerstitial fibrosis are connected with poor prognosis [2]. The deposition

\footnotetext{
* Corresponding author

e-mail: mmcucchiarini@hotmail.com,

ahmadian.elham@yahoo.com
}

of the immune complex at the outer layer of the glomerular basement membrane (GBM), which in turn disturbs the functionality of the glomerular filtration barrier and results in proteinuria has been documented as the main hallmark of MGN [3-5]. This in situ antibody deposition occurs in response to the presence of antigens in podocytes, of which the M-type phospholipase A2 receptor (PLA2R) is the main antigen involved in almost all cases of primary MGN [6].

From a pathologic point of view, diffuse thickening and spike generation in GBM are the major observed alterations. However, such changes are dynamic and might not be distinguished from minimal change disease (MCD) in early stages, therefore other diagnostic techniques such as electron microscopy or immunofluorescence are required [7]. In the absence of fresh tissues and/or specimens without glomeruli 
section, however, these methods are ineffective, and not all clinical centers are equipped with electron microscopy. Activation of the complement system is a critical event in the initiation of several glomerular diseases [8].

The complement factor C5b-9 is found in the urine and renal tissues of MGN patients as a marker of complement system cytotoxicity [9]. In contrast, IgG4 is the chief immunoglobulin deposited in the kidneys of patients with primary MGN [10]. When the classical and lectin pathways of the complement system are activated, the $\mathrm{C} 4 \mathrm{~d}$ fac-tor/component is produced upon the degradation of $\mathrm{C} 4$ and can form a strong covalent bond with the tissue. Therefore, $\mathrm{C} 4 \mathrm{~d}$ has been utilized as a marker of complement system activation and antibody-related response [11]. C4d has also been classically used in the diagnosis of antibody-mediated rejection (AMR) in clinics, but has been implicated in the detection of complement activation and disease prognosis in glomerulopathies [12]. Given the significance of the involvement of the complement system in MGN, the present work is aimed at evaluating the expression of C4d in MGN cases by immunohistochemistry, as well as monitoring its specificity and sensitivity as an important biomarker of the dis-ease.

\section{MATERIALS AND METHODS}

\section{Patients}

In this cross-sectional diagnostic study, of patients referred to the Nephrology Department of the Educational Center Imam Reza Treatment and Research Hospital, 33 patients with glomerulonephritis were included in the patient group. The control group encompassed 20 patients diagnosed with minimal change disease (MCD) with normal histology and without any antibody deposition as noted by immunofluorescence microscopy. All patients provided informed consent before inclusion in the study. Silver jones, hematoxylin and eosin (H\&E), and periodic acid-Schiff (PAS) tests were applied to screen for MGN, and immunofluorescence microscopy as a gold standard technique confirmed the presence of glomerulonephritis.

The immunofluorescence microscopy procedure consisted of the following: $4 \mu \mathrm{m}$-thick frozen sections of the biopsies were fixed in acetone for $5 \mathrm{~min}$ at $-20^{\circ} \mathrm{C}$ followed by incubating in a polyclonal rabbit anti-C4d (Gibco, Invitrogen) for $1 \mathrm{~h}$. Fluorescein isothiocyanate (FITC)-labeled goat anti-rabbit (Sigma) and Texas Red-labeled horse antimouse (Vector) antibodies were then utilized to incubate the slides for half an hour. The diluation employed was $1: 100$, and incubation occurred in a humidified chamber at room temperature, under conditions of darkness. Finally, immunofluorescence microscopy (Olympus,Tokyo, Japan) was applied to visualize $\mathrm{C} 4 \mathrm{~d}$ double-immunofluorescence section slides. All those in the patient group were found to have primary idiopathic MGN.

The inclusion criteria for both groups were as follows: patients with an age range of 14 to 60 years old, IgG and C3 immunofluorescence of at least +2 in the patient group, normal serum creatinine, and standard sample preparation. The presence of other diseases such as lupus, cirrhosis, and $\mathrm{HIV}$, and $<6$ fixed glomeruli were considered as the exclusion criteria. Immunofluorescence was refereed to be positive if there were positive granular capillary staining in the glomeruli, and negative if there were no staining in glomeruli. Each stain was given a score on a scale of $0-4+[13]$. The ethics committee of Tabriz University of Medical Sciences approved this study (IR.TBZMED.REC.1397.1020).

\section{Immunohistochemical evaluation}

The detection of $\mathrm{C} 4 \mathrm{~d}$ via immunohistochemistry was performed on formalin-fixed, paraffin-embedded kidney sections $(4 \mu \mathrm{m})$ through an indirect immunoperoxidase staining method. The activity of endogenous peroxidase in renal tissue sections was prohibited using 8-min 1:4, $30 \%$ hydrogen peroxide dilution with distilled water after the deparaffinization step. The retrieval of the antigen was achieved through a cooking process at $250^{\circ} \mathrm{C}$ under 1 bar pressure for $13 \mathrm{~min}$. Primary polyclonal rabbit IgG antihuman $\mathrm{C} 4 \mathrm{~d}$ antibodies were implemented and an incubation step for $60 \mathrm{~min}$ at $37^{\circ} \mathrm{C}$ took place. Afterward, the secondary antibody, HRP2 (BIO CARE) was utilized and ultimately the slides were tested by two independent pathologists under a light microscope (Olympus BX53, Tokyo, Japan). The immunohistochemistry scoring results for $\mathrm{C} 4 \mathrm{~d}$ were based on the staining intensity of the GBM as follows: $(+1)$ faint reactivity; $(+2) ;(+3) ;(+4)$ strong reactivity [14].

\section{Statistical analysis}

Data obtained from the study were analyzed using the Mann-Whitney test, T-test, and Chi-square test as descriptive statistical methods. The receiver operating characteristic (ROC) curve, which is a graphical plot demonstrating the diagnostic ability of a binary classifier system [15] was plotted to identify the predictive value of $\mathrm{C} 4 \mathrm{~d}$-staining and the sensitivity specificity. Pearson correlation coefficient, and Kappa coefficient were calculated. Spss18 (SPSS, Chicago, IL) was used to analyze the data.

\section{RESULTS}

In this study, 53 patients (33 patients diagnosed as primary MGN versus 20 patients with MCD as a control group) were included. The mean age was $47 \pm 14$ in the target MGN patients and $33 \pm 14$ in the MCD control group, this difference was significant $(\mathrm{P}=0.002)$. The mean proteinuria $(\mathrm{mg} / 24 \mathrm{~h}$ ) level was $3 \mathrm{~g} / 24 \mathrm{~h}$ in MGN patients and 2.9 $\mathrm{g} / 24 \mathrm{~h}$ in the MCD control group $(\mathrm{P}=0.715)$. The creatinine level was considered normal in all patients (mean value of $0.98 \mathrm{mg} / \mathrm{dL}$ ). There was no difference in the serum level of creatinine between the studied groups $(\mathrm{P}=0.082)$ (Table 1).

Table 1. Demographic and clinical data

\begin{tabular}{|c|c|c|c|c|c|}
\hline \multicolumn{2}{|c|}{ Characteristics } & $\begin{array}{l}\text { Total NS } \\
\text { patients }\end{array}$ & MCD group & MGN group & P-value ${ }^{a}$ \\
\hline \multicolumn{2}{|c|}{ No. of cases } & 52 & 20 & 32 & - \\
\hline \multirow{2}{*}{$\begin{array}{l}\text { Gender \% } \\
\text { (N) }\end{array}$} & Male & $53.8(28)$ & $45(9)$ & $59.4(19)$ & \multirow{2}{*}{$0.312^{b}$} \\
\hline & Female & $46.2(24)$ & $55(11)$ & $40.6(13)$ & \\
\hline \multicolumn{2}{|c|}{$\begin{array}{l}\text { Age, mean } \pm \text { SD } \\
\text { (years) }\end{array}$} & $41.71 \pm 15.93$ & $33.15 \pm 14.00$ & $47.06 \pm 14.8$ & 0.002 \\
\hline \multicolumn{2}{|c|}{ Proteinuria (mg/24h) } & $3.09 \pm 1.48$ & $2.95 \pm 1.35$ & $3.09 \pm 1.25$ & 0.715 \\
\hline \multicolumn{2}{|c|}{$\begin{array}{l}\text { Serum creatinine } \\
(\mathrm{mg} / \mathrm{dL})\end{array}$} & $0.98 \pm 0.33$ & $0.88 \pm 0.22$ & $1.00 \pm 0.22$ & 0.082 \\
\hline
\end{tabular}


Immunofluorescence microscopy revealed values of +2 to +4 for IgG and of up to +2 for $\mathrm{C} 3$ in the target MGN patients. In contrast, the results of immunofluorescence microscopy were negative in all MCD patients of the control group (Figure 1).

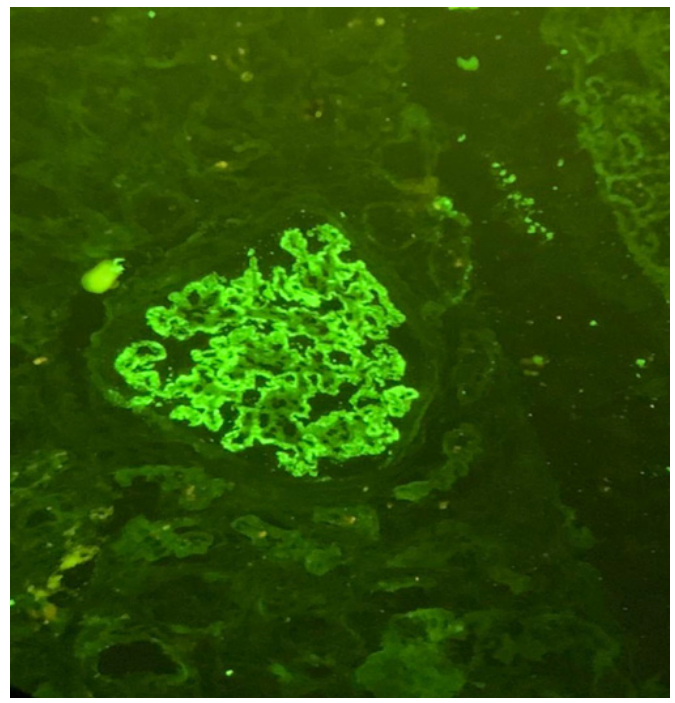

Figure 1. Immunofluorescent staining of C4d complement in MGN. IgG and the complement are accumulated in the glomerular basement membrane and exhibit a diffuse granular pattern

Immunohistochemistry analysis showed an intensity of +2 to +4 for $C 4 d$ in the majority of the MGN patients, while no deposition was observed in the MCD control group (Figure 2).

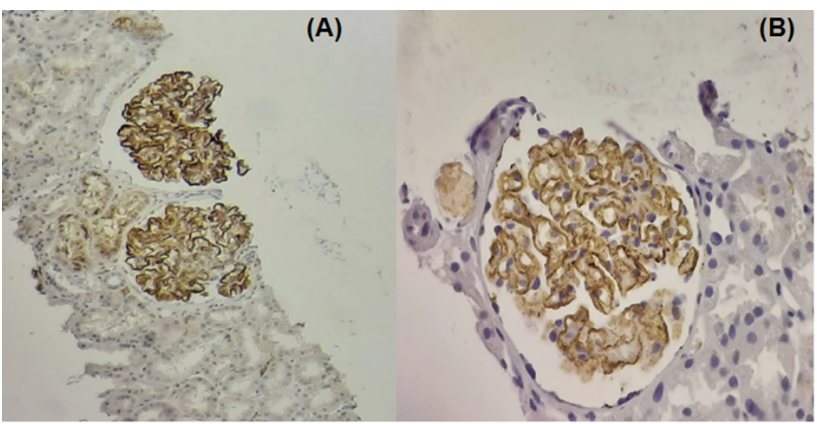

Figure 2. Immunohistochemistry staining of C4d complement product with $\times 10$ (A) and $\times 40$ (B) of Olympus CX23 microscope. The deposition intensity of +3 and +4 is observed in all kidney glomerules

Also, the results of the ROC curve showed a sensitivity of $95 \%$ and specificity of $100 \%$ for C4d (Figure 3).

The Pearson correlation coefficients were 0.74 between C4d (immunohistochemistry) and IgG (immunofluorescence) (Table 2) and 0.65 between C4d and C3 (Table 2), which could be due to the small size of the samples tested, as well as the lower intensity of $\mathrm{C} 3$ in the immunofluorescence test.

Furthermore, the Kappa coefficient values were 0.42 and 0.006, respectively, between C4d (immunohistochemistry) and IgG (immunofluorescence) and C4d and C3 (Table 3).

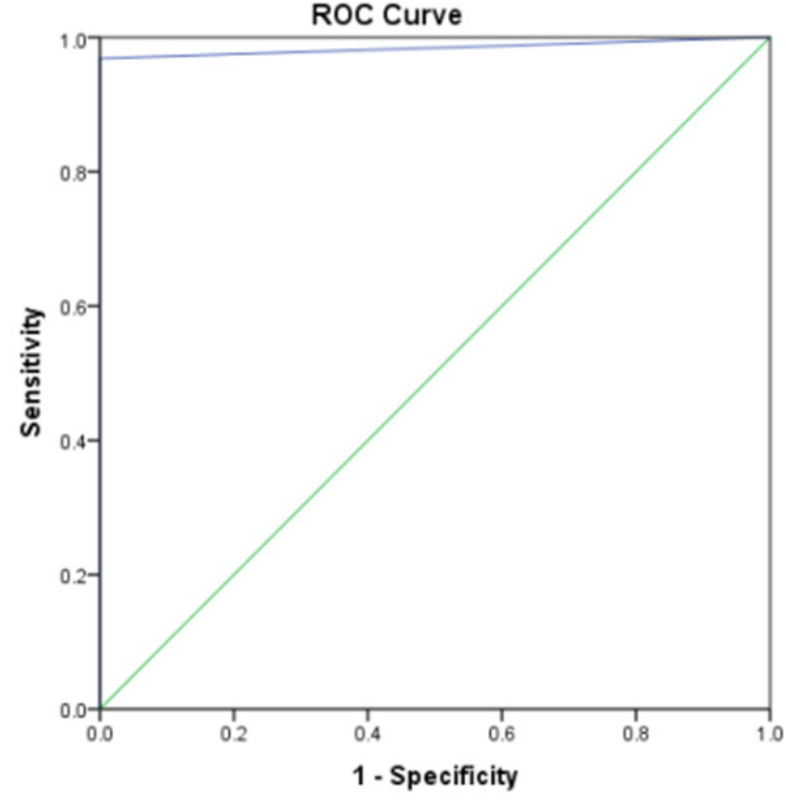

Figure 3. ROC curve for the C4d-staining. The intensity of markers-staining scores was used and the results of IgG and C3 were used as gold standard tests

Table 2. Pearson correlation coefficients between C4d and IgG and between C4d and C3

\begin{tabular}{|l|l|c|c|c|}
\hline \multicolumn{2}{|c|}{ Spearman's rho } & IF IgG & IF C3 & C4d intensity \\
\hline \multirow{2}{*}{ IF IgG } & r & 1 & -0.374 & 0.744 \\
\cline { 2 - 5 } & Sig. (2-tailed) & - & 0.048 & $<0.001$ \\
\hline \multirow{2}{*}{ IF C3 } & r & $-0.374^{*}$ & 1 & -0.091 \\
\cline { 2 - 5 } & Sig. (2-tailed) & 0.048 & - & 0.653 \\
\hline \multirow{2}{*}{ C4d intensity } & r & 0.744 & -0.091 & 1 \\
\cline { 2 - 5 } & Sig. (2-tailed) & $<0.001$ & 0.653 & - \\
\hline \multirow{2}{*}{ IF: immunofluorescent. r: Correlation Coefficient }
\end{tabular}

Table 3. Kappa coefficients between $\mathrm{C} 4 \mathrm{~d}$ and $\operatorname{IgG}$ and between C4d and C3

\begin{tabular}{|l|c|c|c|c|}
\hline & Value & $\begin{array}{c}\text { Asymptotic } \\
\text { Standard Error }\end{array}$ & Approximate Tb & P-value \\
\hline $\begin{array}{l}\text { Measure of Agreement } \\
\text { Kappa between C4d } \\
\text { and IgG }\end{array}$ & 0.426 & 0.116 & 3.912 & $<0.001$ \\
\hline $\begin{array}{l}\text { Measure of Agreement } \\
\text { Kappa between C4d } \\
\text { and C3 }\end{array}$ & 0.006 & 0.006 & 0.416 & 0.678 \\
\hline
\end{tabular}
error assuming the null hypothesis

\section{DISCUSSION}

MGN is a major glomerulopathy that has been extensively investigated from a pathophysiological point of view to develop more efficient treatment opportunities [16]. While numerous studies aimed at defining the target antigen, the current research focused on the effector mechanisms of podocyte damages, especially the complement system activation and the effect of cytokines. The complement system might be activated through three main pathways: (1) the classical C1q pathway; (2) the lectin pathway; (3) the alternative pathway which is connected by the hydrolysis of the C3 segment [17]. The membrane attack complex (C5b-9) is generated after the activation of any of these pathways, resulting in membrane damages of target cells [8]. 
Accumulating evidence from experimental studies indicates a close connection between the activation of the complement system and the progression of glomerular diseases [18]. The deposition of interstitial vascular $\mathrm{C} 4 \mathrm{~d}$ has been reported in acute and chronic AMR [19,20]. Both the classical and lectin complement pathways can result in the formation of inactive $\mathrm{C} 4 \mathrm{~b}$, which then can turn into the surface-bound split $\mathrm{C} 4 \mathrm{~d}$ product. The $\mathrm{C} 4 \mathrm{~d}$ receptor has not been described so far and its biological functions remain unknown [21].

Normal human kidney, as well as primary and secondary nephropathies, has been reported with $\mathrm{C} 4 \mathrm{~d}$-positive staining. The physiological clearance of IgM-compromising immune complexes might be the reason for weak segmental C4d staining in normal renal biopsies [22]. Elevated IgM glomerular deposits have been observed in C4-deficient animals [23]. The podocyte proteins, the PLA2R, are the target antigen in MGN and its antibodies are mostly IgG4 types [24]. The activation of the complement system and in situ immune system reactions are involved in local renal injuries in empirical models [25]. However, the complement system is not activated by $\operatorname{IgG} 4$, therefore, we propose that other IgG subclasses may activate the complement system in MGN. The presence of C4d deposits has been previously documented in MGN samples and is utilized as positive control staining in kidney transplantation in the diagnosis of AMR [26].

Although the pivotal role of the complement system in MGN has been elucidated, information regarding the predominant pathway involved in MGN is still sparse. Previous studies mainly showed more than 905 positive cases regarding C4d in MGN patients [27-29]. The results of Song et al. [30], however, did not indicate the presence of $\mathrm{C} 4 \mathrm{~d}$ in primary MGN, as compared with secondary MGN patients with lupus erythematosus. This might be due to different methodologies applied in different studies. Moreover, the number of patients included in the studies might address such discrepancies.

C1q positive expression has also been reported, along with that of $\mathrm{C} 4 \mathrm{~d}$, suggesting a possible involvement of the classical pathway in this study. The presence of C1q was supported by other studies where the segmental MN pattern was observed [31]. Immunostaining of complement factors showed positive results for factor $\mathrm{B}$, the main marker of the alternative pathway [32]. Recently, the activation of the alternative pathway in a primary $\mathrm{MN}$ case has been documented [33], with intense deposition of factor B, PLA2R, and IgG4, while $\mathrm{C} 1 \mathrm{q}$ and $\mathrm{C} 4 \mathrm{~d}$ exhibited weak deposition. The occurrence of glomerular injuries has been connected with an absence and/or malfunction of counter-regulatory proteins in the alternative pathway as reported in experimental studies [34]. Therefore, a plausible hypothesis is an activation of the classical pathway by $\operatorname{IgG}$ resulting in the stimulation of the alternative pathways and, in particular, the lectin pathway, with time [9]. As supported by different studies, the lectin pathway is the major activated complement system in MN [35]. In a study conducted by Custódio et al. [29], C1g and C4d were investigated in $69 \mathrm{MGN}$ patients, with C4d being positive in $84 \%$ of the cases and $\mathrm{C} 1 \mathrm{~g}$ in only $17 \%$ of them, showing that the lectin pathway may be mostly involved in MN [29]. The presence of other antigens rather than PLA2R might explain the diverseness of the pathways activated in the complement system [36].

For the first time to our best knowledge, our study examined the sensitivity and specificity of $\mathrm{C} 4 \mathrm{~d}$ expression via immunohistochemistry and evaluated its value in the diagnosis of MGN instead of the immunofluorescence technique - which is not available in all centers and requires fresh tissues. According to our results, $95 \%$ sensitivity and $100 \%$ specificity were calculated for the $\mathrm{C} 4 \mathrm{~d}$ marker. In the study from Hai and colleagues [37], the target group included primary and secondary MGN patients and the control group had different diagnoses (including DM, and amyloidosis). Here, C4d immunohistochemical staining was positive in the target patients and negative in the control group.

Secondary MGN in the target group can be a confounding factor in the research results. To omit it here, all the cases were selected from idiopathic primary nephropathy. In line with our work, Hernandez et al. [38] also studied $\mathrm{C} 4 \mathrm{~d}$ deposition in MN patients by immunohistochemistry, but, in marked contrast to our blinded evaluation, the pathologist was not blind regarding the results of optical microscopy and immunofluorescence, which can also be a confounding factor. Beyond the aforementioned, Lim et al. [39] reported that $\mathrm{C} 4 \mathrm{~d}$ deposition in the surrounding capillaries of transplanted MN patients was more elevated than IgG nephropathy post-transplantation, although 19 cases were only examined. Thus, larger sample sizes must be investigated. In another study, C4d deposition was diagnosed in patients with different diseases and the diffuse granular deposition pattern in the glomerular capillaries of $\mathrm{MN}$ was considered as a distinguishing factor [27]. Still, to differentiate primary and secondary $\mathrm{MN}$, again, studies with larger sample sizes will be necessary. In this study, the Pearson and Kappa correlation coefficients were calculated which is essential to determine the diagnostic value of immunohistochemistry relative to other techniques. Overall, and compared with other studies, our data show that the $\mathrm{C} 4 \mathrm{~d}$ deposition in capillary loop glomeruli of MN patients due to the activation of the lectin pathway may be considered as a useful diagnostic tool.

\section{CONCLUSIONS}

Based on this study, immunohistochemical staining of C4d may be used as a surrogate method to diagnose MGN. Investigating the outputs in larger sample sizes is ongoing to provide more accurate and reliable results. To improve the diagnostic value of this approach, the $\mathrm{C} 4 \mathrm{~d}$ deposition pattern with intensity and percentage may also be evaluated in other types of glomerulonephritis.

\section{FUNDING}

This work was financially supported by Tabriz University of Medical Sciences, Tabriz, Iran (grant no. \#60797). 


\section{INSTITUTIONAL REVIEW BOARD STATEMENT}

The ethics committee of Tabriz University of Medical Sciences approved this study (IR.TBZMED.REC.1397.1020).

\section{INFORMED CONSENT STATEMENT}

Informed consent was obtained from all subjects involved in the study.

\section{DATA AVAILABILITY STATEMENT}

Data is contained within the article or supplementary material

\section{ACKNOWLEDGMENTS}

The authors are grateful to the Connective Tissue Diseases Research Center and Kidney Research Center of Tabriz University of Medical Sciences for their kind support. The present work was extracted from the residential thesis of Sonya Sharifi (project number of 60797) at this University.

\section{CONFLICTS OF INTEREST}

The authors declare no conflict of interest.

\section{ORCID iDs}

Magali Cucchiarini (Dhttps://orcid.org/0000-0003-0323-8922

\section{REFERNCES}

1. Dahan K. Membranous nephropathy: diagnosis, new insights in pathophysiology, and therapeutic approach. Rev Med Interne. 2016;37(10):674.

2. Ponticelli C, Passerini P. Can prognostic factors assist therapeutic decisions in idiopathic membranous nephropathy. $J$ Nephrol. 2010;23(2):156-63.

3. Ronco P, Debiec H. Pathophysiology of extramembranous glomerulopathies. Fifty years of progress, from laboratory to patient. Biologie aujourd'hui. 2013;207(4):249-59.

4. Debiec H, Hanoy M, Francois A, Guerrot D, Ferlicot S, Johanet C, et al. Recurrent membranous nephropathy in an allograft caused by IgG3 $\kappa$ targeting the PLA2 receptor. J Am Soc Nephrol. 2012;23(12):1949-54.

5. Filinte D, Arıkan H, Koç M, Kaya H, Özener IC, Akbaş G. The intensity of PLA2R and C4d immunoexpression in primary membranous nephropathy. South Clin Ist Euras. 2020;31(2).

6. Debiec H, Ronco P. Immunopathogenesis of membranous nephropathy: an update. Sem Immunopathol. 2014;36(4):381-97.

7. Torbati PM, Haleh Tavakolian H. Diagostic Accuracy of C4d-IHC in Diagnosis of Membranous Glomerulonephritis. Iran J Kidney Dis. 2020;14(1):20.

8. Kościelska-Kasprzak K, Bartoszek D, Myszka M, Żabińska M, Klinger M. The complement cascade and renal disease. Arch Immunol Ther Exp. 2014;62(1):47-57.

9. Ma H, Sandor DG, Beck LH. The role of complement in membranous nephropathy. Sem Nephrol. 2013;33(6):531-42.

10. Beck LH, Salant DJ. Membranous nephropathy: recent travels and new roads ahead. Kidney Int. 2010;77(9):765-70.

11. Cohen D, Colvin RB., Daha MR, Drachenberg CB. Haas M, Nickeleit $\mathrm{V}$, et al. Pros and cons for $\mathrm{C} 4 \mathrm{~d}$ as a biomarker. Kidney Int. 2012;81(7):628-39.

12. Espinosa M, Ortega R, Sanchez M, Segarra A, Salcedo MT, Gonzalez F, et al. Association of C4d deposition with clinical outcomes in IgA nephropathy. Clin J Am Soc Nephrol. 2014;9(5): 897-904.
13. Larsen C P, Messias NC, Silva FG, Messias E, Walker PD. Determination of primary versus secondary membranous glomerulopathy utilizing phospholipase A2 receptor staining in renal biopsies. Mod Pathol. 2013;26(5):709-15.

14. Stangou MJ, Marinaki S, Papachristou E, Liapis G, Pateinakis P, Gakiopoulou $\mathrm{H}$, et al. Histological grading in primary membranous nephropathy is essential for clinical management and predicts outcome of patients. Histopathology. 2019;75(5):660-71.

15. McClish DK. Analyzing a portion of the ROC curve. Med Decis Making. 1989;9(3):190-5.

16. Glassock RJ. The pathogenesis of idiopathic membranous nephropathy: a 50-year odyssey. Am J Kidney Dis. 2010;56(1):157-67.

17. Noris M, Remuzzi G. Overview of complement activation and regulation. Semin Nephrol. 2013;33(6):479-92.

18. Sheerm NS, Risley P, Abe K, Tang Z, Wong W, Lin T, et al. Synthesis of complement protein $\mathrm{C} 3$ in the kidney is an important mediator of local tissue injury. FASEB J. 2008;22(4):1065-72.

19. Colvin RB. Pathology of chronic humoral rejection. Contrib Nephrol. 2009;162:75-86.

20. Einecke GB, Sis J, Reeve M, Mengel PM, Campbell LG, Hidalgo B, et al. Halloran antibody-mediated microcirculation injury is the major cause of late kidney transplant failure. Am J Transplant. 2009;9(11):2520-31.

21. Murata K, Baldwin WM. Mechanisms of complement activation, C4d deposition, and their contribution to the pathogenesis of antibodymediated rejection. Transpl Rev. 2009;23(3):139-50.

22. Zwirner J, Felber E, Herzog V, Riethmüller G, Feucht HE. Classical pathway of complement activation in normal and diseased human glomeruli. Kidney Int. 1989;36(6):1069-77.

23. Zwirner J, Felber E, Burger R, Bitter-Suermann D, Riethmüller G, Feucht HE. Classical pathway of complement activation in mammalian kidneys. Immunology. 1993;80(2):162.

24. Beck Jr LH, Bonegio RGB, Lambeau G, Beck DM, Powell DW, Cummins TD, et al. Salant M-type phospholipase A2 receptor as target antigen in idiopathic membranous nephropathy. New Engl J Med. 2009;361(1):11-21.

25. Couser WG. Primary membranous nephropathy. CJASN. 2017;12(6):983-97.

26. Ranjan P, Nada R, Jha V, Sakhuja V, Joshi K. The role of C4d immunostaining in the evaluation of the causes of renal allograft dysfunction. Nephrol Dial Transplant. 2008;23(5):1735-41.

27. Val-Bernal J, Fernando M, Garijo F, Val D, Rodrigo E, Arias M. C4d immunohistochemical staining is a sensitive method to confirm immunoreactant deposition in formalin-fixed paraffin-embedded tissue in membranous glomerulonephritis. Histol Histopathol. 2011;26(11):1391-7.

28. Espinosa-Hernández M, Ortega-Salas R, López-Andreu M, Gómez-Carrasco JM, Pérez-Sáez MJ, Pérez-Seoane C, et al. C4d as a diagnostic tool in membranous nephropathy. Nefrología (English Edition). 2012;32(4):536.

29. Bichuette CF, Aparecida da Silva C, Rodrigues Helmo F, Reis Machado J, Antônia dos Reis M. Complement System and C4d expression in cases of Membranous nephropathy. Braz J Nephrology. 2017;39(4):370-5.

30. Song YS, Min KW, Kim JH, Kim GH, Park MH. Differential diagnosis of lupus and primary membranous nephropathies by IgG subclass analysis. Clin J Am Soc Nephrol. 2012;7(12):1947-55.

31. Obana M, Nakanishi K, Sako M, Yata N, Nozu K, Tanaka R, et al. Segmental membranous glomerulonephritis in children: comparison with global membranous glomerulonephritis. Clin J Am Soc Nephrol. 2006;1(4):723-9.

32. Segawa Y, Hisano S, Matsushita M, Fujita T, Hirose S, Takeshita M, et al. IgG subclasses and complement pathway in segmental and global membranous nephropathy. Pediat Nephrol. 2010;25(6):1091-9.

33. Bally S, Debiec H, Ponard D, Dijoud F, Rendu J, Fauré J, et al. Phospholipase A2 receptor-related membranous nephropathy and mannan-binding lectin deficiency. J Am Soc Nephrol. 2016;27(12):3539-44.

34. Cunningham PN, Quigg RJ. Contrasting roles of complement activation and its regulation in membranous nephropathy. J Am Soc Nephrol. 2005;16(5):1214-22. 
35. Ponticelli C, Glassock RJ. Glomerular diseases: membranous nephropathy -a modern view. Clin J Am Soc Nephrol. 2014;9(3): 609-16.

36. Murtas C, Bruschi M, Candiano G, Moroni G, Magistroni R, Magnano A, et al. Coexistence of different circulating anti-podocyte antibodies in membranous nephropathy. Clin J Am Soc Nephrol. 2012;7(9):1394-400.

37. mmunohistochemistry in membranous nephropathy. J Lab Physicians. 2014;6(2):76.
38. Espinosa-Hernández M, Ortega-Salas R, López-Andreu M, GómezCarrasco JM, Pérez-Sáez MJ, Pérez-Seoane C, Aljama-García P. C4d as a diagnostic tool in membranous nephropathy. Nefrología (English Edition). 2012;32(3):295-9.

39. Lim BJ, Kim MS, Kim YS, Kim SI, Jeong HI. C4d deposition and multilayering of peritubular capillary basement membrane in posttransplantation membranous nephropathy indicate its association with antibody-mediated injury. Transplant Proc. 2012;44(3):619-20. 